\title{
Carnets
}

Revue électronique d'études françaises de l'APEF

Deuxième série - 8 | 2016

Du Français en cause aux causes du français

\section{Penser en français}

\section{Cristina Robalo Cordeiro}

\section{OpenEdition \\ Journals}

Édition électronique

URL : http://journals.openedition.org/carnets/1820

DOI : $10.4000 /$ carnets. 1820

ISSN : 1646-7698

Éditeur

APEF

\section{Référence électronique}

Cristina Robalo Cordeiro, «Penser en français », Carnets [En ligne], Deuxième série - 8 | 2016, mis en ligne le 30 novembre 2016, consulté le 23 avril 2019. URL : http://journals.openedition.org/ carnets/1820; DOI : 10.4000/carnets.1820

Ce document a été généré automatiquement le 23 avril 2019.

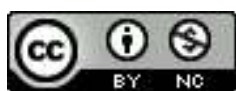

Carnets est mis à disposition selon les termes de la licence Creative Commons - Atribution - Pas d'utilisation commerciale 4.0 International. 


\title{
Penser en français
}

\author{
Cristina Robalo Cordeiro
}

1 En donnant comme titre à cette intervention «Penser en français ", j'ai le sentiment de m'écarter sans doute à l'excès des questions qui ont jusqu'à présent été agitées dans les diverses prises de parole, comme de celles qui font mon quotidien professionnel.

2 Je vis dans la gestion, dans l'administration, dans la machinerie de la francophonie universitaire. L'AUF, vous le savez, est un vaste réseau, reliant plus de 800 universités de par le monde : le Maghreb représente à lui tout seul près de $15 \%$ de cet univers et cette puissance numérique lui a permis de faire élire le premier président maghrébin de l'Agence Universitaire de la Francophonie. C'est assez dire que les forces géopolitiques sont décisives et que c'est à grande échelle, à l'échelle démographique d'abord, qu'il faut en effet envisager le problème linguistique. L'AUF est née de cette prise de conscience de l'importance du Nombre. La partie ne sera pas perdue tant que nous saurons nous appuyer sur une portion, de plus en plus instruite, de la jeunesse de la planète. L'avenir $\mathrm{du}$ français passe donc par la jeunesse africaine mais il est vrai aussi que l'avenir de l'Afrique passe par le français, autant que par l'anglais et le portugais (et certainement pas par le chinois) : d'où l'importance de bien calculer, d'investir à bon escient, dans un esprit de conquête - ou de reconquête - méthodique en tenant compte de l'ensemble des déterminants économiques, sociologiques, culturels et cultuels. Ainsi les mots " stratégies ", " planification », « enjeux », " programmes ", " projets ", " mise en place », « veille » ... composent à peu près tout le lexique dont j'ai besoin dans mes échanges avec mes semblables. C'est une combinatoire très limitée dont on a vite épuisé tous les agencements possibles. Et pour une ancienne professeure de littérature, c'est, je le reconnais, une purge très amère dont le seul mérite subjectif est d'aiguiser mon appétit de libre réflexion.

Voilà pourquoi j'ai choisi de me rapprocher ce matin du cœur de la question du français en délaissant un moment ce qui en fait le squelette externe. Car, après tout, une langue naturelle, une langue historique c'est une affaire de cœur, une affaire de vécu. Et s'il y a des langues qui donnent peu de latitude à l'expression de la personne, à l'affirmation de l'autonomie individuelle, le français, plus qu'aucune autre peut-être, pour des raisons d'histoire idéologique, est une langue du Sujet et, plus précisément, du Sujet pensant. 
4 Non pas que le français soit une langue spécialement vouée, par sa morphologie, à la philosophie. Comme dit Caetano Veloso « Il est prouvé qu'il n'est possible de philosopher qu'en allemand» (Língua, chanson du disque Velô). Il reste que c'est en français que Descartes a écrit : «Je pense donc je suis », en français et pas même en latin (le «Cogito ergo sum » n'est qu'une traduction postérieure due à Etienne de Courcelles). Or la pensée n'est pas le privilège des philosophes de profession, des techniciens du concept. Et c'est justement ce qu'a voulu rappeler Descartes en adressant son Discours de la Méthode non pas aux docteurs de la Sorbonne mais au plus large public possible, y compris aux femmes, comme une espèce de manifeste démocratique.

5 Avant de revenir sur cette foi dans l'aptitude universelle au raisonnement - « le bon sens est la chose du monde la mieux partagée » - et sur la façon de conduire ses idées que la langue française, telle qu'elle existe aujourd'hui, semble favoriser, je vais m'arrêter un instant sur le premier degré de la réflexion : celui de la pensée parlée.

6 Il y a là un ensemble de points qui intéressent directement la pédagogie de la langue. J'ai bien conscience de manquer absolument de prudence en m'avançant sur ce terrain que la plupart d'entre vous connaissent beaucoup mieux que moi, en particulier ceux qui sont versés dans les sciences cognitives. Mais, en prenant aujourd'hui des leçons d'initiation à la langue arabe (à vrai dire de darija) il m'est permis, je crois, de formuler des propos relativement ingénus - et certainement anachroniques - que me suggère ce retour à l'école dans la situation de l'apprenant.

7 Voici donc la première question : que veut dire « penser dans la langue étrangère " pour un débutant ou même pour tout locuteur qui n'est pas parfaitement bilingue ? Pas grandchose sans doute. C'est pourtant un conseil souvent donné dans les cours de langue et je le donnais moi-même quand il m'est arrivé de dispenser des cours de langue française au collège : « Oubliez le portugais, pensez directement en français !» La démarche naturelle, tenue pour fautive, consiste: 1 , à concevoir l'idée; 2 , à l'énoncer dans sa langue ; 3 , à traduire cet énoncé dans la langue étrangère. Il faut remplacer cette procédure complexe et artificielle par une immersion immédiate dans la langue étrangère. Autrement dit, la langue étrangère doit cesser d'être un point d'arrivée pour être un point de départ. Il y a là un défi apparent à la logique, et qui est cependant le paradoxe fondateur de toute méthode directe : commencer par la fin, parler d'abord et penser ensuite. Car penser en parlant n'est possible que si on a "rendu l'initiative aux mots» disait un illustre professeur d'anglais : Stéphane Mallarmé. Et c'est avec des mots qu'on fait des syntagmes, éventuellement des poèmes, encore plus qu'avec des idées.

Dresser l'automate, comme le voulait le behaviorisme, construire des réflexes, comme le voulait Pavlov, il ne semble pas qu'il y ait pour l'acquisition d'une langue vivante d'autre base possible. L'acte d'intellection y a peu de part et il est souhaitable qu'il soit réduit au minimum. Ce n'est pas autrement que, d'après Eugen Herrigel, l'on apprend la technique du tir à l'arc dans le bouddhisme Zen (Herrigel, 2007). La réflexion, si elle intervient, sera peut-être la récompense, mais en aucun cas le moyen du progrès.

Demandant un jour à un collègue linguiste allemand pourquoi, à son avis, les étudiants allemands semblaient dominer mieux que les autres les langues étrangères, il me répondit que leurs premiers efforts portaient sur le rudiment phonétique et que l'élève ne pouvait pas passer à l'étape phonologique tant qu'il ne maîtrisait pas la prononciation. Blaise Pascal, de son côté, reconnaissait la primauté de ce qu'il appelait «la machine » même en matière de religion. «Abêtissez-vous! » : la formule a fait scandale. Elle signifie 
seulement que la spiritualité viendra remplir des formes vides mais que ces formes vides doivent lui préexister.

L'apprentissage d'une langue étrangère n'est donc en rien comparable à celui des autres disciplines scolaires, ne pouvant être rapproché qu'avec le cours de musique ou de gymnastique. Et c'est pourquoi nous continuons à nous heurter à des obstacles surgis de notre indéracinable intellectualisme, à commencer par celui des élèves eux-mêmes. Mais - et c'est là où je veux en venir - était-il pour autant nécessaire que la plupart des manuels de langue - en l'occurrence de français - que j'utilisais s'obstinassent à humilier l'intelligence et le bon goût? Je veux croire que, depuis que j'ai cessé d'enseigner la langue française - voici vingt ans - vous avez vu apparaître des ouvrages - quel qu'en soit le support - capables de satisfaire à la fois aux besoins spécifiques du cours de langue et à ceux, plus généraux, de l'esprit, lequel demande à être respecté et nourri dans chaque élève, qu'il soit petit ou grand débutant.

11 Je veux le croire et j'en suis du reste convaincue : l'apparition des arts dans les cours de langues (comme en témoigne le programme de ce colloque) prouve que la didactique du FLE a compris tous les bénéfices qu'on peut escompter d'une alliance avec l'esthétique comme elle sait tirer parti des études culturelles. Le magnifique livre d'hommage à Clara Ferrão Tavares n'aurait pas été possible ni même concevable dans les années 80 de l'autre siècle : la didactique vivait alors encore des années de plomb. Il est aujourd'hui permis à l'élève et au professeur de français de penser en parlant. De penser, au sens où Descartes lui-même entendait le mot, c'est-à-dire de sentir et d'imaginer. "Plus rien d'oiseux ou simplement de gratuit » comme le recommandait Mallarmé qui voulait qu'« un seul et même esprit, littéraire autant que pratique " préside à l'usage de la méthode qu'il proposait (Mallarmé, 1974 : 1058).

Pour résumer ce premier point, je dirai que " penser en parlant » veut dire deux choses bien distinctes. Si penser signifie raisonner, il importe que le débutant s'abstienne de le faire. Confions-nous à l'automate. Répétons et apprenons par cœur. C'est à quoi je m'efforce dans mes leçons de darija. Mais si penser veut dire sentir et imaginer, je ne vois pas en quoi la poésie, la peinture et la musique ne seraient pas d'efficaces auxiliaires du cours de langue. En bref pourquoi un manuel pour débutant ne lui permettrait pas de cueillir la "fleur même " de la langue (c'est encore une expression de Mallarmé), autrement dit les cultures qui l'illustrent? Ce qui nous semble aujourd'hui une évidence, n'oublions jamais qu'il a fallu un demi-siècle de recherches en didactique pour le découvrir.

13 J'ai laissé soigneusement de côté la question très obscure de savoir si la subjectivation des idées, ou l'intériorité pensante, met en jeu le langage. Les uns, la plupart, diront que oui : le langage est toujours déjà là : penser c'est parler ou se parler. Les autres diront que non : pour eux, au commencement n'est pas le verbe, mais le geste, le mouvement, le corps luimême rythmant, articulant la pensée inchoative, comme chez l'animal ou le jeune enfant (c'est la thèse de Bergson dans Matière et Mémoire). Ce qui est hors de doute c'est que la pensée humaine se structure dans le langage et que le langage humain ou disons, pour parler comme André Martinet, "la double articulation» prend toujours la forme singulière d'une langue.

14 Je n'ai pas, Dieu merci, besoin de voir très clair sur ce point pour accéder au deuxième degré de ma spéculation. Si je me suis d'abord demandé ce que peut vouloir dire pour l'élève débutant de français " penser en parlant » - et mon résultat a été plutôt négatif ou au moins limitatif - je vais maintenant me placer devant une nouvelle question en 
accompagnant l'élève dans son développement: penser en français, c'est entrer dans l'esprit de la langue par la lecture, c'est penser en lisant. Quelle découverte l'attend ? Je mets le mot « découverte » au singulier car il ne s'agit pas de la myriade d'informations relatives à la diversité sensible de la culture, voire des cultures que véhicule en propre la langue française, mais bien plutôt de l'essence ou, plus modestement, de la tendance essentielle du français.

Je suppose, pour simplifier les choses, que mon élève, intelligent, curieux, avide de lecture, est de langue portugaise. Tout le travail de pensée de ce disciple idéal va consister à comprendre qu'il lui faut passer d'une langue-culture d'inspiration baroque à une langue-culture d'inspiration classique. C'est vite dit, et il faut toute une vie de bilingue pour en prendre acte! D'origine latine tous les deux, les langues portugaise et française entretiennent un rapport de symétrie inversée, qui procure chacune à la pensée une expansion de sens opposé. Seule une longue pratique de lecteur, et mieux encore de traducteur, apportera la vérification de cet écart ou mieux de cette antithèse opposant les deux langues, qu'éclairent leurs histoires culturelles et politiques autant que strictement linguistiques.

Ayant assez de lucidité pour ne pas me lancer, devant des spécialistes, dans une comparaison sommaire entre les deux systèmes ni même entre les deux évolutions, j'abriterai mes remarques sous l'autorité du Vocabulaire européen des philosophies, ouvrage monumental élaboré sous la direction de Barbara Cassin (2004). L'article signé par Alain Badiou, professeur à l'ENS-Ulm, cherche à caractériser le français tel que l'écrivent les penseurs français depuis Descartes. L'article signé par Fernando Santoro, professeur à la Fédérale de Rio, fait de même pour le portugais, depuis le Padre Vieira. Je ne saurais trop vous inviter à prendre connaissance de ces deux remarquables études qui, en quelques pages, dégagent deux climats spirituels où croissent, à partir de nos deux langues, des types de végétations philosophiques apparemment incompatibles entre elles. Le contraste est d'autant plus saisissant que les deux auteurs ont écrit leurs articles indépendamment l'un de l'autre.

17 Faute de temps pour les résumer, je me contenterai d'en extraire trois oppositions qui, pour être presque banales, n'en sont pas moins pertinentes: d'un côté, nous avons le portugais, «langue baroque ", qui porte la pensée à la luxuriance, à l'ambiguïté, au sensible. De l'autre, le français, "langue classique " (au sens évidemment français du terme, c'est-à-dire anti-baroque), qui appelle à la sobriété, à l'univocité, à l'abstraction. D'un côté, l'exubérance tropicale, les jeux de miroir, l'ici et le maintenant du « estar », de l'autre, la rigueur géométrique, la précision juridique, l'intemporalité de l'idée générale. En somme le Padre Vieira contre le chevalier Descartes, le Sermão da sexagésima contre le Discours de la Méthode. Et le plus fort que c'est trois siècles plus tard nous retrouvons chez Pessoa et chez Valéry, par exemple, les mêmes tendances antagoniques, comme si, décidément, le même esprit s'obstinait à habiter chacune des langues en déterminant un style de penser inaliénable.

18 Nous le savons, surtout quand nous traduisons, le français est une "langue maigre» (Badiou parle du français comme "évidement», le portugais une langue pléthorique (Santoro évoque notre penchant immodéré pour la redondance). Je ne me priverai pas du plaisir de reproduire l'exemple lumineux donné par le professeur de Rio (Cassin, 2004 : 974). Le philosophe brésilien, bilingue, Emmanuel Carneiro Leão écrit dans «Terceiro Mundo », p. 4, la phrase suivante : «Todo passo é uma aventura de originalidade : passeando 
pela essência do real, nossos passos caminham pela originalidade do caminho, caminhar $e$ caminhada ».

Que devient ce petit texte, jouant sur l'étymologie et la paronymie, une fois traduit en français par l'auteur lui-même? Voici :

Car chaque pas est une aventure. En se promenant à travers l'essence de la réalité, nos pas cheminent dans l'originalité du chemin.

Traduit à la lettre, le portugais ferait l'effet d'un insupportable galimatias. Il faut donc absolument émonder, choisir, aplanir et, assurément, appauvrir la pensée pour qu'elle soit lisible en français. C'est que la langue française, après Rabelais et Montaigne, est passée par les mains chirurgicales de Vaugelas, de Malherbe, des grammairiens des salons précieux, des Messieurs de Port-Royal, des Académiciens de Richelieu pour en sortir, svelte et nerveuse, prête à se jeter dans les bras de Racine... Mais avant Racine, il y avait eu La Rochefoucauld et Pascal qui avaient mis au point l'art de la concision et de la frappe, que nous retrouvons intact au $20^{\mathrm{e}}$ siècle chez Barthes ou chez Cioran.

Penser en lisant les auteurs français, ce sera donc toujours, qu'on soit chez Voltaire ou chez Rousseau, chez Lacan ou chez Deleuze, si différents entre eux ou même si hostiles les uns aux autres, se soumettre à la phrase française, que Badiou n'a pas tout à fait tort de comparer au lit de Procuste (Cassin, 2004 : 472). Aussi peut-on affirmer que toute pensée française aspire à s'enfermer dans un aphorisme, c'est-à-dire dans une formule péremptoire et définitive. Cette prédilection pour la maxime fait que les Français sont en définitive davantage des «moralistes » que des métaphysiciens.

Mais il est temps que j'aborde le troisième degré de l'apprentissage. Si mon élève s'est montré aussi patient que vous et que je le tienne encore sous ma férule, je voudrais lui montrer ce qu'il doit savoir faire pour penser à la française quand il écrit.

23 Je ne manque jamais l'occasion de faire l'éloge de l'exercice scolaire de dissertation et je n'hésite pas à déclarer qu'il fait gravement défaut dans notre enseignement national. Pour en apprécier tout l'intérêt il faut, me dit-on, l'avoir longuement pratiqué au collège et à l'université. J'évoquais tout à l'heure l'art du tir à l'arc, je pourrais aussi bien me référer à l'art équestre dont seule une longue pratique permet la compréhension intime.

Le mot dissertation est sans doute trompeur puisque nous parlons en portugais de « dissertação » à propos de nos thèses de doctorat, ce qui ne viendrait jamais à l'esprit d'un Français. Je ne vous ferai pas l'injure de vous expliquer en quoi consiste l'entrainement dialectique auquel sont soumis les candidats au baccalauréat et, plus encore, les candidats aux grandes écoles, y compris scientifiques. Très rares du reste sont ceux qui dominent la technique car il n'est certes pas facile d'improviser, en quatre ou six heures, sur un sujet imposé, un système cohérent et, idéalement, semblable à une petite mécanique bien huilée. Mais rien de tel non plus pour encourager l'intelligence à prendre l'initiative, à mobiliser ses ressources propres, à esquiver les objections.

Je me souviens d'avoir fait l'essai de cette formation spéciale sur des étudiantes de mestrado en les mettant au défi d'élaborer des "plans » de commentaire composé : après une phase d'instruction où il a fallu contrarier d'anciennes habitudes mentales, elles se sont tellement prises au jeu que notre séminaire s'est transformé en concours d'agilité mentale, parfois sophistique il est vrai - mais la sophistique ne mérite pas sa mauvaise réputation.

$\mathrm{Au}$ demeurant, l'épreuve de dissertation ne manque pas d'adversaires et il n'est pas certain qu'elle survive encore longtemps à la massification de l'enseignement secondaire. 
Elle aura pourtant aidé à former des générations de brillants penseurs : Lévi-Strauss, Derrida, Foucault - pour ne citer que des noms mythiques, ont tous été dressés à cette école du raisonnement, assez analogue à une préparation militaire de l'esprit. Et si cette image vous surprend, je peux la justifier en remontant aux origines du mouvement de réforme qui a introduit la dissertation littéraire dans les lycées au moment où, dans les années 1880, la IIIe République refondait l'université française. Voici ce qu'écrit l'historien Joseph Hours :

Après les désastres de 1870, la nation avait entrepris son examen de conscience. Elle avait été à peu près unanime à attribuer sa défaite à un manque de méthode et de réflexion, à l'habitude de suivre au hasard les impulsions du sentiment. Tout cela, disait Flaubert, c'était arrivé "parce qu'on n'avait pas lu L'Education sentimentale ». Il en était résulté un désir passionné de travail intellectuel, d'action vigoureuse dirigée par une pensée ferme selon des méthodes scientifiques et d'après les enseignements de l'expérience. Tous les partis s'étaient efforcés de satisfaire ce désir, mais les républicains y étaient arrivés mieux que leurs adversaires... (Hours, 1945 : 293).

Avant cette époque, l'exercice traditionnel dans les classes était le "discours français » qui incitait l'élève à faire preuve d'éloquence en utilisant toute la gamme des figures de rhétorique. Mais la terrible défaite devant l'armée de Bismarck et les grandes convulsions qui en ont suivi sont venues dénoncer la vanité de cette éducation oratoire et plus encore les dangers du verbalisme romantique. Il était urgent d'adopter un modèle agonistique de formation intellectuelle et d'armer les enfants de la bourgeoisie de l'équipement dialectique dont leurs parents étaient dépourvus. Il convient de noter que l'influence d'Emmanuel Kant, dont les détracteurs qualifiaient la doctrine de «caporalisme prussien » a été très forte sur les maîtres de l'enseignement français qui donnaient volontiers comme exemple parfait de progression discursive le plan tripartite des Fondements de la Métaphysique des Mours (Kant, 1994)ํ․

Ainsi les Français, dans les vingt dernières années du XIX siècle se guérissaient finalement $d u$ " mal du siècle » en réapprenant à penser à l'école de l'Allemagne. Mais ils apportaient à cette méthodologie leur caractère national : le refus du jargon technique, le désir de plaire autant que de convaincre, le souci de ne pas séparer la philosophie de l'écriture littéraire. Henri Bergson, si imprégné de kantisme, réalise le type même du penseur français, se voulant à la fois profond et artiste, clair et vigoureux. Il est significatif qu'il soit redevenu aujourd'hui une référence majeure, à un moment où la philosophie veut se rapprocher du grand public.

"Langue des femmes et des prolétaires plutôt que des savants ", comme dit Alain Badiou (Cassin, 2004: 465), le français philosophique, qui s'en était, dans une certaine mesure, éloigné sous le romantisme (mais Stendhal et Balzac sont philosophes), a envahi la littérature depuis plus d'un siècle : A la Recherche du Temps perdu est-elle une philosophie romancée ou un roman philosophique ? Camus, Sartre, Simone de Beauvoir bien sûr, mais plus près de nous combien d'agrégé(e)s de philosophie se cachent parmi les romanciers et romancières à la mode ! Agrégés de philosophie c'est-à-dire, dans le contexte français, des virtuoses de la dissertation. Non pas qu'un bon roman soit une dissertation réussie - loin de là ! - mais il y a malgré tout de l'exercice académique à la construction d'une fiction une continuité latente, celle de la langue française elle-même avec son formalisme immanent et sa syntaxe impérieuse. A la fin de bon nombre de romans français, le lecteur pourrait écrire : «Ce qui devait être démontré a été démontré ». Et tout cela est arrivé parce que, finalement, on a lu L'Education sentimentale... 
Vous pouvez, avec justesse, me reprocher d'avoir prononcé un plaidoyer pour l'intelligence française et vous auriez beau jeu de citer mille exemples de la bêtise des Français. Mais où, mieux que dans ces Assises, faire l'apologie d'une certaine manière de conduire sa raison et de chercher la vérité, sinon dans les sciences, comme l'espérait Descartes ${ }^{2}$, en tout cas dans les lettres, où la vérité ne se distingue pas de l'adhésion qu'on cherche à obtenir de son public?

\section{BIBLIOGRAPHIE}

CASSIN, Barbara (dir) (2004). Vocabulaire européen des philosophies. Paris : Seuil / Le Robert.

HERRIGEL, Eugen (2007). Zen e a arte do tiro com arco. Lisboa : Biblioteca editores Independentes.

HOURS, Joseph (1945). Cuvre et pensée du peuple français. Paris : Librairie Bloud \& Gay.

KANT, Emmanuel (1994). Fondements de la Métaphysique des Mœurs. Paris : Flammarion.

MALLARMÉ, Stéphane (1974). Thèmes anglais, in Cuvres complètes. Paris : Gallimard, « Bibliothèque de la Pléiade ».

\section{NOTES}

1. Première section : Passage de la connaissance rationnelle commune de la moralité à la connaissance philosophique. Deuxième section : Passage de la philosophie morale populaire à la métaphysique des mœurs. Troisième section: Passage de la métaphysique des mœurs à la critique de la raison pure pratique.

2. Discours de la méthode pour bien conduire sa raison et chercher la vérité dans les sciences.

\section{RÉSUMÉS}

Si l'on tente de saisir la spécificité de la langue française, il peut être utile d'écarter un instant les nombres et les espaces qui déterminent sa dimension quantitative pour se concentrer sur le Sujet parlant. Dans une comparaison avec le portugais, nous décrivons ici ce que signifie 1. penser en parlant, 2. penser en lisant, 3. penser en écrivant.

Trying to grasp the specificity of the French language, it may be useful to forget for a moment the numbers and spaces which determine its quantitative dimension and to concentrate on the speaking Subject. In a comparison with the Portuguese, we describe here what it means 1 . to think while speaking, 2. to think while reading, 3. to think while writing... in French. 
INDEX

Mots-clés : sujet, exercice, sophistique, intelligence, automate

Keywords : subject, exercise, sophistic, intellect, automat

\section{AUTEUR}

CRISTINA ROBALO CORDEIRO

Universidade de Coimbra

crobalo[at]uc.pt 